\title{
HACIA UNA EVOLUCIÓN EN EL CAMPO DEL CONOCIMIENTO DE LA DISCIPLINA ADMINISTRATIVA: DE LA ADMINISTRACIÓN DE EMPRESAS A LA GESTIÓN DE ORGANIZACIONES*
}

\author{
PAULA ANDREA LÓPEZ"* \& ANDRÉS MARIÑO ARÉVALO \\ FUNDACIÓN UNIVERSIDAD CENTRAL DE COLOMBIA
}

Recibido/ Received/ Recebido: 17/09/2010 - Aceptado/ Accepted / Aprovado: 15/11/2010

\begin{abstract}
Resumen
En este artículo se pretende reflexionar sobre las condiciones actuales de la disciplina administrativa, así como sobre los retos a los que se enfrenta en aras de responder a la evolución que en los últimos años ha tenido. En ese sentido, es necesario superar la visión enfocada en la Administración de Empresas y centrarse en la discusión sobre la Gestión de Organizaciones, lo que ineludiblemente implica un paso de la visión mecanicista, reduccionista y lineal imperante, hacia un enfoque de integración disciplinar bajo el paradigma de la complejidad .
\end{abstract}

Palabras clave: Disciplina administrativa, Administración, Gestión, Organizaciones, Complejidad.

\section{TURNING TO BUSINESS MANAGEMENT TO THE MANAGEMENT OF ORGANIZATIONS: THE EVOLUTION OF THE KNOWLEDGE OF THE MANAGEMENT DISCIPLINE}

\begin{abstract}
This article reflects on the current conditions of administrative discipline, as well as the challenges it faces in order to respond to developments in recent years has had. In that sense, it is necessary to overcome the vision focused on Business Administration and focus in the discussion on the Management of Organizations, which inevitably involves a step of the mechanistic view, the prevailing reductionist and linear, toward an integrative approach to discipline under paradigm of complexity. Key words: Administrative discipline, Administration, Management, Organizations, Complexity.
\end{abstract}

Este artículo es producto de la investigación realizada para la creación del programa académico Maestría en Gestión de Organizaciones de la Universidad Central, a cargo del grupo de investigación GIGO-UC.

* Administradora de Empresas. Universidad Nacional de Colombia. Magíster en Administración. Universidad Nacional de Colombia. Estudiante de Doctorado en Ciencias Económicas, Universidad Nacional. Docente Investigadora. Grupo de Investigación GIGOUC. Universidad Central. plopezl@ucentral.edu.co

*** Administrador de Empresas. Universidad Nacional de Colombia. Magíster en Administración. Universidad Nacional de Colombia. Docente Investigador. Grupo de Investigación GIGO-UC. Universidad Central. amarinoa@ucentral.edu.co 


\title{
TRANSFORMANDO A GESTÃO DE NEGÓCIOS PARA A GESTÃO DAS ORGANIZAÇÕES: A EVOLUÇÃO DO CONHECIMENTO DISCIPLINAR
}

\begin{abstract}
Resumo
Neste artigo vamos refletir sobre as condições atuais de disciplina administrativa, bem como os desafios que enfrenta, a fim de responder à evolução nos últimos anos tem tido. Nesse sentido, é necessário superar a visão focada em Business Administration e foco na discussão sobre a gestão das organizações, o que inevitavelmente envolve uma etapa da visão mecanicista, o reducionista predominante e linear, em direção a uma abordagem integrada para a disciplina em paradigma da complexidade.
\end{abstract}

Palavras-chave: Disciplina de Administração, Administração, Gestão, Organizações, Complexidade.

López, P. \& Mariño, A. (2010) Hacia una evolución en el campo del conocimiento de la disciplina administrativa: De la administración de empresas a la gestión de organizaciones. En: Revista de la Facultad de Ciencias Económicas de la Universidad Militar Nueva Granada. rev.fac.cienc.econ, XVIII (2)

JEL: M10.

\section{Introducción}

La disciplina administrativa en los últimos años, ha estado sometida a constantes exámenes y revisiones, por parte de académicos, empresarios, expertos, etc., que analizan su pertinencia en el afán por generar condiciones sociales, económicas, culturales y políticas adecuadas, que le permitan a los seres humanos que conforman las organizaciones desarrollarse como tal, y a su vez posibilitar la construcción de una sociedad más equitativa y justa. Para esto, ha sido necesario reflexionar sobre el cuerpo teórico que ha influido en la forma en la cual se han abordado, comprendido, intervenido y por ende gestionado las organizaciones.

Este artículo se fundamenta en los aspectos teóricos que de alguna manera han guiado la Administración y que más recientemente guían la Gestión de Organizaciones. En este sentido, la construcción del documento atiende a los elementos necesarios para abordar la evolución desde la administración hasta la gestión, considerando aspectos que permiten también realizar una aproximación a la evolución en su objeto de estudio. En consecuencia, es preciso reflexionar desde diferentes perspectivas, enfo- ques y conceptos que le den pertinencia al discurso emergente en la disciplina Administrativa.

El artículo está estructurado en tres partes. La primera presenta la discusión que se ha dado frente a los conceptos Administración y Gestión con el propósito de situar la postura de los autores en este sentido. En la segunda parte se relaciona el estado de discusión de la gestión señalando el paradigma tradicional que ha dominado el discurso, el paradigma emergente, así como una nueva perspectiva que está en proceso de construcción denominada Ciencias de la Gestión. En la tercera parte se presenta una conceptualización sobre organizaciones, su comprensión desde la complejidad, así como la propuesta en torno a que significa una gestión compleja. Finalmente, se presentan una reflexión con el ánimo de señalar la necesidad de superar la visión enfocada en la Administración de Empresas y centrarse en la discusión sobre la Gestión de Organizaciones; lo que ineludiblemente implica un paso de la visión mecanicista, reduccionista, y lineal imperante hacia un enfoque de integración disciplinar, que permite una mejor comprensión del fenómeno organizacional bajo el abrigo del paradigma de la complejidad. 


\section{Discusión alrededor de la Administración y la Gestión}

En la disciplina administrativa es frecuente encontrar el uso indistinto de las palabras administración y gestión, lo que hace difícil identificar una diferenciación clara entre los términos, incluso son considerados de alguna manera sinónimos. Sin embargo, más allá de la consideración puramente semántica, se podría precisar que dentro de las lógicas de la organización, la administración y la gestión tienen objetos de intervención diferentes, con procesos y procedimientos particulares que hace que se diferencien tanto en sus propósitos como en sus alcances. A continuación se presenta un intento por establecer la distinción entre administración y gestión. Una mirada etimológica de los términos Administración y Gestión nos conduce a entender porque la cercanía en la definición de los términos.

Tabla 1. Conceptos de Administración y Gestión ${ }^{1}$

\begin{tabular}{|c|c|c|}
\hline $\begin{array}{l}\text { ADMINISTRACIÓN/ } \\
\text { ADMINISTRATION }\end{array}$ & MANAGEMENT & GESTIÓN \\
\hline $\begin{array}{l}\text { La raíz de los términos administrer y } \\
\text { administration, es totalmente latina, } \\
\text { proveniente del verbo administrare, } \\
\text { que puede definirse directamente } \\
\text { como administrar un bien, adminis- } \\
\text { trar en defensa de los intereses de } \\
\text { quienes nos confían su patrimonio } \\
\text { (Aktouf, 2003). }\end{array}$ & $\begin{array}{l}\text { La palabra management procede del latin manus, } \\
\text { que significa mano, vocablo del cual procede el } \\
\text { verbo inglés to mange, que significa inicialmente } \\
\text { entrenar, hacer, andar, conducir un caballo (Aktouf, } \\
\text { 2003). De manera reciente se relaciona con mane- } \\
\text { jar, conducir, gobernar, dirigir, administrar. De allí } \\
\text { surge Management, el cual ha sido traducido en el } \\
\text { idioma inglés como administración, organización, } \\
\text { supervisión y en otros idiomas como gerencia, go- } \\
\text { bierno, gestión, administración, grupo que decide, } \\
\text { maneja recursos, dirige empresas, negocios (Mar- } \\
\text { tínez, 1999, p.29). }\end{array}$ & $\begin{array}{l}\text { Gere y gestión, provienen del verbo } \\
\text { latino genere, que significa condu- } \\
\text { cir (en el sentido amplio de llevar o } \\
\text { llevar a cabo) y dirigir e incluso go- } \\
\text { bernar (Aktouf, 2003). }\end{array}$ \\
\hline
\end{tabular}

Como se puede ver, desde lo etimológico, los términos Administración y Gestión están vinculados con la noción de conducción y dirección. Sin embargo, existen diferencias profundas entre los términos si la mirada se hace desde su concepción y los procesos de intervención en las organizaciones, es en este sentido, en que se valida la diferencia estructural entre Administración y Gestión.

En cuanto al concepto de administración, es necesario señalar su significativa evolución, desde inicios del siglo $\mathrm{XX}^{2}$ donde se empieza a construir su marco de referencia, fundamentalmente enmarcado en las propuestas de Taylor y Fayol, como exponentes de la Administración Científica y la Administración Industrial respectivamente. Quienes plantea- ron conceptos de Administración en ambos casos, centrados en unos principios. Para Taylor, la Administración se enmarca en un conjunto de principios encaminados a lograr la máxima productividad y la prosperidad para el trabajador. Por su parte Fayol, asume la Administración como un conjunto de principios que se desarrollan bajo la previsión, la organización, la dirección, la coordinación y el control.

H. Simon (1972, 240), plantea una noción más amplia de administración, centrando su atención en el proceso de toma de decisiones:

"Relacionadas con el colectivo humano que conforma la organización, en función de los objetivos a alcanzar, en sus

Fuente: Elaboración de autores con base en Aktouf (2003), Martínez (1999).

2 Aunque los vestigios de la administración datan de la antigüedad, se puede establecer que el discurso administrativo se empezó a formalizar como tal, desde los planteamientos realizados en 1903 por Taylor. 
diferentes fases: formulación del problema, análisis, síntesis y determinación de alternativas, incluida la definición de criterios de elección, su implementación y evaluación".

Para Peter Drucker $(1975,18)$, la administración se define como una:

"Nueva función social, motor de cambio y desarrollo, ligada a variables como la cultura organizacional, el espíritu empresarial, la innovación social y la creatividad".

Ampliando el concepto tradicional que se tenía de administración. Aktouf (2003, 17), logra una definición que da cuenta del desarrollo histórico del concepto, la define como una:

"Serie de actividades interdependientes destinadas a lograr que cierta combinación de medios (financieros, humanos, etc.) pueda generar una producción de bienes o servicios económicos o socialmente útiles y en lo posible rentables para la empresa con fines de lucro".

Los diferentes conceptos presentados, aunque no agotan las propuestas que en este sentido se han generado, sí permiten visualizar la evolución que ha tenido el concepto de Administración, incluso las diversas nociones que han sido desarrolladas en diferentes contextos y que responden a condiciones políticas, económicas, sociales, culturales particulares, y muestran el avance que han tenido en cuanto a disciplina.

En lo que se refiere al concepto Gestión, bien se puede plantear que aún es un concepto en construcción en la disciplina administrativa, se encuentra en un proceso de consolidación de un cuerpo teórico solido que desde dicha perspectiva permita el entendimiento de los fenómenos propios de la disciplina. La gestión se ha entendido coloquialmente como el "hacer diario de los directivos". Etimológicamente, gestión viene de gerere y significa llevar a cabo, y en el siglo XIX hacía referencia a la acción de hacer diligencias. Por su parte, Aktouf, señala que geres y gestión provienen del verbo latino gerere, que ya en la lengua de Cicerón significaba conducir (...) y dirigir, e incluso, gobernar (Aktouf, 1998, 18). Lorino por ejemplo $(1995,2)$ plantea que la gestión es:

"La medida y el análisis, la visión y la comprensión, para la acción. La medida y el análisis son la base de la acción, puesto que la acción se hace muy incierta si no se puede ver o no se puede comprender".

La generalidad de las definiciones propuestas alrededor de la gestión, se relacionan con el concepto de administración, son pocas las acepciones que no evocan actividades propias de la administración. Pero desde la lógica que se entiende Gestión en este artículo, se relaciona con la acción dentro de las organizaciones que permite establecer las directrices que las conducen a un estado superior de acuerdo con su identidad, fines y propósitos. Esto evidencia que el concepto de gestión, plantea una visión más amplia que lo estrictamente operacional. Para llevarla a cabo se requiere de conocimientos, conceptos y herramientas que conducen a la articulación de los subsistemas de la organización. En consecuencia:

"La gestión evoca la estrategia [...] y hoy se considera una construcción nueva [...] que se coloca en el plano de intermediar entre las grandes y gruesas directrices del manejo económico y resolver la dinámica del funcionamiento de las organizaciones. La gestión no existe en sí misma, es el producto de la interacción de múltiples actores, los cuales acaban determinando un sistema de manejo, de proyección y de visión el cotidiano y del futuro" (Romero, 1998, 35).

La gestión permite proyectar la organización con los componentes que el entorno le ofrece, apoyándose para ello en la estrategia, la cual se constituye en su eje, porque permite establecer el camino para construir el futuro de la organización. La gestión no solamente se centra en el manejo de lo inter- 
no, sino también de los elementos determinantes del entorno. Así la gestión cumple una función de articulación entre la organización y los demás sistemas del medio y asume la labor de proyección de la organización, para lo cual debe estar en contacto continuo con las variables externas.

Adicionalmente, como mecanismo de regulación, la gestión se asegura que los medios utilizados obedezcan a los fines, permitiendo así que la organización reflexione sobre su propio comportamiento y sea capaz de modificarlo si es necesario. Es decir, la gestión es esencialmente acción sobre la organización, acción del latín actionem; que significa toda manifestación de intención o expresión de interés capaz de influir en una situación dada.

La acción en la gestión se ve materializada por la estrategia, lo que genera una relación recursiva entre ambos conceptos. Dicha relación se debe abordar desde los diferentes problemas ligados a la acción, que se ha centrado en el estudio de cuatro problemas principales: la perspectiva teleológica, la sociológica, la ideológica y la ecológica. La primera de ellas pone el énfasis en los objetivos de la organización, en su concepción y la forma como la organización los alcanza en el tiempo; la segunda se centra en la comprensión de los diferentes actores que hacen parte del proceso estratégico, tanto internos como externos, quienes formulan las estrategias y llevan a cabo acciones que de ellas se derivan; la tercera, en el espíritu del discurso estratégico, y su influencia, tanto en el orden organizacional como en la sociedad en general; y la cuarta, en los elementos del entorno como el mercado, o los grupos externos de interés, y más recientemente en los elementos como el medio ambiente natural y su relación con la organización.

Pensar entonces, que la administración y la gestión responden a la misma naturaleza y tienen procesos de acción similares en la organización, sería desconocer la evolución de la disciplina y por tanto las implicaciones que este hecho tiene para la comprensión e intervención del fenómeno organizacional. En la evolución de la disciplina, la noción de administración se conoce primero que la noción de gestión.
"La administración ligada más a los orígenes de la formalización inicial de la dirección del trabajo manufacturero, evoca más la destreza de manejar y dirigir el trabajo de otros y a las habilidades de comprensión y aplicación de ciertas técnicas operacionales" (Romero, 1998, 35).

Y la gestión relacionada con los procesos estratégicos, de direccionamiento y proyección de la organización. La gestión, entonces, es considerada dentro del fenómeno organizacional, como un proceso que se da a su interior $y$, que responde a un nivel diferente al de la administración. Y es entendida como: "La acción social de regulación del comportamiento de una colectividad social, provista de un conjunto de recursos de diversa índole, todos ellos limitados en el tiempo, el espacio, la magnitud y la significancia, conducentes al logro de construcciones nuevas y diferentes a la definición inicial [...] es agenciada por individuos que regularmente convocan a los participantes del proyecto que se ha emprendido $y$ que regularmente, aún si éste se les ha impuesto, es el pivote de la acción colectiva compartida o no" (Romero, 1998, 36).

En ese sentido se concibe la gestión como un mecanismo de direccionamiento, mediante el cual se puede orientar el comportamiento de los miembros de la organización hacia el logro de los propósitos estratégicos, que solo se pueden alcanzar a partir de la acción colectiva. Esto evidencia que el concepto de gestión, plantea una visión más amplia que lo estrictamente operacional y más abstracta que la administración. De acuerdo con Sanabria (2007, 167), la gestión:

\section{"Consiste en una actividad transversal a la organización que puede ser desarro- llada de diversos modos y grados, en sus diferentes ámbitos y subsistemas".}

Y va más allá, al afirmar que se debe hablar de tipos particulares de gestión para cada tipo de organización, evidenciando así el alcance más amplio que plantea la gestión frente al concepto de administración, que más que oponerse, se complementan, pues resulta imposible pensar en una gestión de las 
organizaciones que no pueda ser materializada a través de la administración.

La diferenciación entre administración y gestión se considera un proceso que aún se está construyendo, pero cada vez más se reconoce la pertinencia de establecerla. Bien podría decirse que la diferencia fundamental, se encuentra en la proyección que se hace de la organización por medio de la gestión, pero que requiere de la administración (rutinas, tareas operativas) para alcanzarla. La Administración y la Gestión son entonces conceptos complementarios, no excluyentes, es decir, no basta con administrar tareas hay que proyectar la organización con los componentes que el entorno le ofrece, apoyándose para ello en la estrategia y la prospectiva como elementos constitutivos de la gestión de organizaciones.

Tras la conceptualización dada en torno a las definiciones de gestión y administración, se concluye que resulta mucho más amplio y pertinente acoger el concepto de gestión, pues se trata de un concepto transversal a toda la organización, y que recoge a la totalidad de los subsistemas que la componen. Además, se puede relacionar la gestión con los diferentes tipos de organizaciones, siendo influenciada por un observador particular, que a su vez, se sirve de la administración para poder materializar la gestión.

\section{Paradigmas en la Gestión}

Una de las preocupaciones centrales, es la de identificar los aspectos que ha direccionado el discurso actual de la gestión. Lo que obliga a la revisión de los paradigmas que influencian la construcción del discurso en torno a la disciplina Administrativa.

Es pertinente para el propósito de ésta parte del documento establecer elementos conceptuales a los cuales se hará alusión. En este sentido, paradigma es entendido a partir de la propuesta de Morin $(2001,89)$, quien establece que un paradigma:

"Está constituido por un cierto tipo de relación extremadamente fuerte entre nociones maestras, nociones clave, principios clave. Esa relación y esos princi- pios van a gobernar todos los discursos que obedecen, inconscientemente, a su gobierno".

Desde esta postura serán entendidos los paradigmas que han permeado la gestión y que de alguna manera han determinado no solo la forma de concebir el objeto de estudio sino también las formas de aproximación al entendimiento del mismo. De igual manera se presentaran los paradigmas emergentes o nuevos paradigmas que desafían a la gestión, que como bien lo menciona Kuhn (1962), son paradigmas que se enfrentan a la "terquedad" de las estructuras de pensamiento anteriores, que desean permanecer como esquemas de referencia.

La gestión ha estado enmarcada por distintos paradigmas, los cuales han respondido de alguna manera a las circunstancias de orden social, económico, político y cultural que se han generado en distintos momentos en la disciplina. A continuación se presenta el paradigma tradicional que ha dominado el discurso de la gestión desde sus inicios, y el paradigma emergente que surge como respuesta a las nuevas perspectivas y retos a los que se enfrenta la gestión de organizaciones.

\subsection{Paradigma tradicional}

El paradigma tradicional que ha guiado el desarrollo de la Administración, se presenta a partir del planteamiento propuesto por Lorino (1995), quién establece que a la mecánica económica neo-clásica responde la empresa-maquina de los tayloristas, donde la eficiencia, la estabilidad y el control se constituyen en el modelo determinístico de gestión imperante.

Lo anterior es señalado de manera particular en los siguientes cuatro principios (Lorino, 1995, 8-9).

- Principio No. 1. Estabilidad: Los mecanismos de eficiencia, los conocimientos operativos que permiten ser eficientes, son estables en el tiempo. Este principio justifica la noción de los estándares, norma de eficiencia relativamente fijada en el tiempo, y funda todas las formas de gestión en el retrovisor, las que se basan en referencias del pasado. 
- Principio No. 2. Información Perfecta: El directivo (gestor, empresario, decisor) tiene en los mecanismos de la eficiencia del sistema que dirige, una información perfecta.

- Principio No. 3. La eficiencia productiva ${ }^{3}$ : se identifica con la minimización de los costos. Desinteresarse del valor para concentrarse en los costos permite dejar de lado la incertidumbre nacida de los desfases en el tiempo entre la contratación de los recursos (costos) y la realización de las ganancias (valor). Solo se trabaja sobre lo seguro, lo positivo y no sobre lo hipotético.

- Principio No. 4. El costo global es equivalente al costo de un factor de producción dominante. Existe un recurso patrón (ej. mano de obra directa) que sirve de base a los procedimientos de asignación y a la medida del conjunto de los costos.

De esta manera Lorino establece la forma como el pensamiento tradicional, sistemático y formalizado en gestión, opera al abrigo del paradigma dominante de organización perfecta, de cuantificación escalar, de divisibilidad, de atemporalidad, de repetitividad y de estabilidad. En una palabra, una mecánica racional en perfecta coherencia con la epistemología clásica, ésta gestión podrá durablemente, y a excepción de cualquier otra, adornarse con los hábitos de la ciencia, mientras que sus principios fundamentales reciben los primeros ataques debidos a la resistencia de los problemas reales y a los pensamientos que vienen del exterior.

En esta misma medida y en los últimos años ha tomado relevancia para la gestión, el paradigma del mercado, como vector que determina y condiciona las relaciones de las organizaciones. A este respecto Martinet (1990) señala que estrictamente desde el punto de vista del mercado, se muestra su ineptitud para guiar la gestión de una organización, dado que la convierte en una actividad eminentemente instrumental, con un solo foco de atención, por tanto limita su acción de proyección que en esencia la debe caracterizar.

Resultado de dicha concepción tradicional, se han generado distintos focos de atención en la disciplina Administrativa. Cada uno de los autores que se ha acercado a su estudio ha entendido desde su propia perspectiva cuál es el objeto de estudio e intervención de la Administración (el control, las áreas funcionales, la toma de decisiones, el individuo, el grupo, la estructura, el entorno y sus componentes, la cultura, por mencionar algunos de los focos de atención propuestos). En consecuencia sus aproximaciones suelen ser limitadas, solo dan cuenta de la comprensión parcial del objeto, por tanto, no se tienen presente consideraciones amplias y profundas para su abordaje. Esa forma de concebir el objeto, comprenderlo e intervenirlo, permiten evidenciar la perspectiva simple, parcializada, desarticulada y limitada que ha imperado en la concepción del fenómeno organizacional. Hecho que no ha permitido una visión integradora, más bien ha generado una visión reduccionista de la organización y de la gestión. Es decir, los abordajes que ha constituido el discurso responden a fragmentos desarticulados del problema central, se hace referencia al hecho de que la complejidad del fenómeno organizacional no puede ser elucidada estudiando separadamente el control del proceso de producción, las funciones, el problema de la racionalidad, la decisión, el individuo, del grupo, la estructura, el entorno y sus componentes, la cultura entre otros aspectos.

Bien se podría decir, que esta forma tradicional de concebir la gestión se enmarca en el conocido Paradigma de la Simplicidad, que es un paradigma que pone orden en el universo, y persigue el desorden; ve a lo uno y ve lo múltiple, pero no puede ver que lo uno puede, al mismo tiempo, ser múltiple. La simplicidad separa lo que está ligado (disyunción), o bien unifica lo que es diverso (reducción) y estas dos lógicas (disyunción y reducción) son mutilantes. Desafortunadamente desde esta perspectiva se ha construido el discurso administrativo, visión que aún sigue imperante y que genera resistencia a la

3 Tanto si se trata de producir bienes como servicios. 
construcción de una visión compleja de su objeto de estudio.

\subsection{Un paradigma emergente}

La evolución que ha tenido la disciplina administrativa y los entornos regidos por la inestabilidad y la incertidumbre en los que se encuentran inmersas las organizaciones, conducen a la búsqueda de una nueva forma de aproximarse e intervenir el fenómeno organizacional. De tal manera que surge el paradigma de la complejidad como respuesta a los nuevos retos y necesidades, en oposición a una visión mutilante, desarticulada y simplista, dado que la perspectiva de complejidad permite tener una mirada amplia, articuladora e integradora de la organización por ende de su gestión.

Para una mayor claridad, frente a la propuesta de dicho enfoque proporciona a la gestión, se presenta una aproximación a los aspectos que conforman el paradigma de la complejidad.

\subsection{1. ¿Qué es la complejidad?}

La generación de conocimiento desde el S. XVII, ha sido influenciado por el cartesianismo, es decir, operado por la selección de datos significativos y rechazo de aquellos que 'no lo son', que se da a través de la separación y la unión, la jerarquización y la centralización, configurando así el conocido Método Científico. Siendo éste "el imperio de los principios de disyunción, reducción y abstracción, cuyo conjunto constituye el 'paradigma de simplificación'", desarticulando, de acuerdo con este mismo autor, al sujeto pensante y a la cosa extensa, es decir, la filosofía y la ciencia.

Sin embargo, no se trata de emitir un juicio acerca de lo 'bueno' o lo 'malo' que pueda haber en dicha propuesta, pues el mismo Morin afirma que éste ha permitido, sin duda, los enormes progresos del conocimiento científico y de la reflexión filosófica, pero a su vez, también ha limitado el pensamiento. Por esto, en las condiciones actuales se puede considerar que dichas prácticas son insuficientes, pues no basta solamente con ir de lo complejo a lo simple, y asumir que:
"La realidad se puede desarmar para estudiar el comportamiento de las partes por separado, y considerar que es posible luego proceder a reconstruir la conducta global a partir de las partes" (Etkin y Schvarstein, 2005, 83).

Eso llevaría a admitir que un conjunto, puede ser explicado a través de las propiedades de sus componentes individuales.

De allí la necesidad de una propuesta alternativa, pues:

"Mientras que la ciencia de inspiración cartesiana iba muy lógicamente de los complejo a lo simple, el pensamiento científico contemporáneo intenta leer la complejidad de lo real bajo la apariencia simple de los fenómenos. De hecho, no hay fenómeno simple" (Morin, 2007, 56).

Se evidencia entonces la necesidad de avanzar en el paradigma caracterizado (limitado quizás) por la disyunción, la reducción y la abstracción, por un paradigma que incluya, además, la distinción y la conjunción:

"Que permita distinguir sin desarticular, asociar sin identificar o reducir" (Morin, 2001, 34).

Es decir, se hace necesario estructurar una visión compleja para la generación de conocimiento, y superar por esta vía las carencias actuales.

En primer lugar es importante establecer, que no hay acuerdo en la definición del vocablo complejidad. De hecho, cada una de las definiciones proporcionadas se centra en una característica diferente de la complejidad, quedando claro que se trata de un concepto multidisciplinar. Es indudable que la complejidad acerca las disciplinas científica y filosófica, en contraposición a la desarticulación entre sujeto pensante y cosa extensa anteriormente reseñada, rehusando parcelar los problemas fundamentales comunes en todas las disciplinas (Maldonado, 2008; Morin, 2001; Olmedo et al., 2005). Incluso Maldonado $(2003,143)$ va más allá, al afirmar que: 
"Las ciencias de la complejidad redefinen de raíz las relaciones mismas entre las ciencias y la filosofía, así como entre las ciencias entre sí, y por consiguiente, entre ciencia y sociedad".

Lo cual da cuenta del replanteamiento que se da, y de la aparición de parámetros diferentes para la generación del conocimiento, frente a los propuestos anteriormente por el cartesianismo.

Desde la perspectiva de Morin (2001, 32), la complejidad se define como:

"Un tejido (complexus: lo que está tejido en conjunto) de constituyentes heterogéneos inseparablemente asociados: presenta la paradoja de lo uno y lo múltiple [...] es, efectivamente, el tejido de eventos, acciones, interacciones, retroacciones, determinaciones, azares, que constituyen nuestro mundo fenoménico".

La complejidad no es solamente un fenómeno empírico que se justificaría por la constatación del azar, de desordenes, de no acabamientos, de interdependencias en el seno de un fenómeno. Es también un problema conceptual y lógico que rompe las distinciones tradicionales entre conceptos tales como "producto" y "productor", "causa" y "efecto", "uno" y "múltiple", "comienzo" y "fin", "autonomía" y "dependencia", "orden" y "desorden", "sujeto" y "objeto". La complejidad considera en efecto que los procesos de la dinámica de la organización de lo social están asegurados por un conjunto de fuerzas contradictorias y complementarias que se ejercen en el sistema y sobre él, rompiendo así con la habitual visión de la complejidad entendida:

"Como una cuestión de número, de muchos elementos a considerar, centrándose también en los aspectos de relación entre partes del sistema que queremos caracterizar como complejo" (Navarro, 2002, 83).

El paradigma de la complejidad proporciona el marco conceptual para el pensamiento complejo (Del- gado et. al, 2006; Maldonado, 2003; Morin, 2001, 2007). El pensamiento complejo implica otro modo de concebir y pensar el universo que no busca complicar, sino abrir la mente hacia otros conceptos $y$ progresar hacia la comprensión de lo complejo. Y esta comprensión de lo complejo implica aceptar la ambigüedad, la contradicción, la no trivialidad, la policausalidad, la orientación hacia la acción, la falta de precisión, y la impredecibilidad, entre muchas otras.

El concepto de no trivialidad reúne en sí mismo, muchos de los otros aspectos mencionados como la ambigüedad, la contradicción, e incluso, la impredicibilidad. A través de una analogía, Morin (2001) habla de una 'máquina trivial' como aquella de la que al conocer todos sus inputs (entradas), conocemos automáticamente todos sus outputs (salidas), por lo que haría que todo sistema, fuese totalmente predecible. Todo sistema, al menos todo aquel que está influido por un entorno social es, en esencia, impredecible. A pesar de que es la vida social la que lleva a generar un comportamiento de 'máquina trivial', es la misma vida social, a través de sus particularidades (momentos de crisis, decisión, etc.) que asoma lo no trivial, y por tanto, la incertidumbre.

Sin embargo, la incertidumbre no hace su aparición únicamente por la no predictibilidad de los fenómenos, sino porque la visión compleja de estos, debe apartarse de una concepción de causalidad lineal, como la única de las causalidades existentes. Surge entonces el concepto de policausalidad, entendiendo que cada situación no es el simple resultado de una determinada variable de entrada, sino que sobre cada situación, influyen muchas otras variables, que pueden ser dependientes o independientes entre sí, pero que finalmente terminan afectando el resultado (Etkin, 2005). Con esto queda claro que la incertidumbre, no solo hace referencia a los resultados que se pueden dar, sino a las causas que originan dichos resultados, en unas condiciones de espacio y tiempo particulares. A pesar de esto, la complejidad no se ofrece como una receta para conocer lo inesperado (Morin, 2001, 117), pues:

"El pensamiento complejo no rechaza, de ninguna manera, a la claridad, el 
orden, el determinismo. Pero los sabe insuficientes, sabe que no podemos programar el descubrimiento, el conocimiento, ni la acción".

Para poder tener una mejor comprensión de la complejidad, se establece que existen tres principios fundamentales que ayudan a pensar la complejidad (Morin, 2001, 105-107):

a) El principio dialógico: Se expresa en el reconocimiento de la asociación de nociones contradictorias que forman parte del mismo fenómeno. La convivencia del orden y el desorden en constante contradicción, son dos enemigos: uno suprime al otro pero, al mismo tiempo, en ciertos casos, colaboran y producen la organización y la complejidad. Como bien lo señala Etkin (2003) una relación dialógica significa que en el mismo ambiente actúan fuerzas que son complementarias, antagónicas y concurrentes.

b) El principio de recursividad: Es un proceso en el cual los productos y los efectos son al mismo tiempo, causas y productores de aquello que los produce. Se rompe la relación lineal entre causas y consecuencias, existiendo relaciones recursivas entre ambas.

c) El principio hologramático: Establece que en el menor punto de la imagen del holograma contiene la casi totalidad de la información del objeto representado. No solamente la parte está en el todo, sino que el todo está en la parte. Supera el reduccionismo, que sólo se centra en las partes, así como el holismo, que únicamente se centra en el todo. Se basa en suponer que no sólo las partes están en el todo, sino también el todo en las partes.

\subsection{Ciencias de Gestión}

En el campo de la disciplina administrativa desde hace algunos años ha surgido una corriente que busca atender algunos aspectos sobre sus fundamentos epistemológicos, en el intento de constituir una base sobre la cual soportar la disciplina. Los cuestionamientos van orientados a establecer ¿Cuál es su objeto de estudio?, ¿Cómo se constituye el conocimiento formal en Gestión?, ¿Cuáles son las condiciones en que surge dicho conocimiento?, ¿Cuál es su naturaleza y alcance?, ¿De qué métodos hace uso?, ¿Cómo se reconoce socialmente el aporte del conocimiento que produce?

Esta reflexión ha sido objeto de múltiples abordajes, que van desde aproximaciones al conocimiento en gestión con parámetros científicos, en otros casos disciplinares hasta miradas desde lo técnico, e incluso empírico. Desde la mirada científica, hoy se reconoce una corriente de autores que han planteado la posibilidad de hablar de Ciencias de Gestión, como una forma de reconocer la evolución de la disciplina y por tanto de otorgarle un status epistemológico (David et. al., 2000; Pérez, 1998; Martinet, 1990; Le Moigne, 1997).

Lo primero para señalar es que las ciencias de gestión se encuentran en proceso de construcción, y en este proceso se han nutrido de múltiples ciencias y disciplinas para su consolidación, entre las cuales se puede mencionar el derecho, la ingeniería, la economía, la psicología, la sociología, las matemáticas (métodos cuantitativos), la antropología y la ciencia política. Situación que de alguna manera ha puesto en cuestionamiento su propio cuerpo teórico y evidencia la necesidad, que por lo tanto existe, de apelar a una epistemología que explique y valide los nuevos desarrollos que se generan desde dichas ciencias (Romero, 1998). La gestión, se sustenta en aspectos de otras disciplinas por lo cual, sufre muy seguido las quejas, las obstrucciones y negaciones de las otras ciencias sociales, porque no puede ser interrogada a partir de criterios científicos o de pertinencia que le sean propios (David et al, 2000).

Las ciencias de gestión son las más jóvenes de las ciencias sociales. Se han beneficiado en alguna medida del reconocimiento que ha adquirido la organización como un actor productor de sociedad. Pero su reconocimiento no se traduce aún con mucha claridad sobre el plano científico. Es en esta área donde las evoluciones son largas y complejas, las ciencias de la gestión sufren siempre de un déficit de identidad: ellas deben mostrar que tienen un objetivo propio (David et al, 2000). 
Para Pérez (1998), las ciencias de gestión encuentran su posicionamiento científico según su grado de implicación y de aplicación, se interesan en las organizaciones en general, al provenir de las ciencias humanas y sociales y buscan no solamente analizar las organizaciones, sino también mejorar su funcionamiento, lo que las conduce a ser disciplinas "profesionalizantes". A partir de esto Pérez, plantea que las ciencias de gestión son a la vez científicas, tecnológicas y profesionales:

- Científicas: Porque reposan sobre una vena de conocimientos nutrida por la investigación.

- Tecnológicas: Porque implica un cierta cantidad de "Saber-Hacer", en unión con los procedimientos y/o los equipamientos específicos.

- Profesionales: Porque busca preparar en determinados oficios.

Sin embargo, para David, Hatchuel \& Laufer (2000) las ciencias de gestión desembocan hoy en una situación que ellas no podían imaginar claramente en sus inicios: una ciencia cuyo objetivo no sería ni un tipo de organización, ni un tipo de fenómenos, ni un conjunto de hechos, pero si más bien una clase de problemáticas constitutivas de toda acción colectiva: la decisión, la racionalización, la representación, la legitimidad, la cooperación, la prescripción. Por lo tanto, las ciencias de gestión son entonces inevitablemente las ciencias de colectivos capaces de transformar y que piensan que esa transformación pasa necesariamente como nuevos conocimientos y nuevas formas de acción en las organizaciones sociales.

Las ciencias de gestión entonces:

\begin{abstract}
"Deben propender por un acercamiento entre la teoría y el pragmatismo, pero esto no significa una fusión, incluso se puede decir que las dos, teoría y práctica, cohabitaran en una relación mixta de lucha y de cooperación" (Martinet, 1990, 17).
\end{abstract}

En el proceso de construcción de su identidad. De la construcción de la identidad de las ciencias de gestión, Le Moigne $(1997,164)$ señala que:

\begin{abstract}
"Algunos defensores de la disciplina prefieren retroceder prudentemente $y$ presentarla como una técnica o un arte; prudencia nociva puesto que no admite que la gestión sea objeto de la ciencia $y$, por tanto, merecedora de atención epistemológica. Otros, más numerosos, proponen una salida más audaz: las ciencias de gestión son "una disciplina donde convergen otras disciplinas". ¿Su cientificidad es, en ese caso, la de sus componentes? Pero las ciencias de gestión no son disciplinas parroquiales, son autónomas, tan legítimas a priori como la balística, o la geografía. Y aceptando que este hecho es un dato observado y por tanto 'positivo', entonces lo que hay que preguntarse es sobre la naturaleza de sus fundamentos epistemológicos".
\end{abstract}

Para Le Moigne (1997) si la epistemología que fundamenta aparentemente a las ciencias de gestión parece incoherente $y$, no obstante, la disciplina exhibe la vitalidad suficiente para que las comunidades científicas no la consideren charlatanería, cabe preguntar cuál es el origen de esa falta de confianza: ¿la disciplina no es compatible con la epistemología que usa como referencia, o el referente epistemológico no es compatible con la disciplina?". En consecuencia, en vez de partir de los postulados básicos del positivismo para evaluar la cientificidad de los enunciados producidos por las ciencias de gestión, se debe partir de otro referente epistemológico que pueda admitir su vitalidad.

En esta misma dirección, Martinet (1990) señala entonces, la factibilidad de una epistemología endógena de las ciencias de gestión, y se basa en los planteamientos de Piaget (1975) para exponer que el nuevo hecho en las disciplinas, es su capacidad para que la reflexión epistemológica se haga desde dentro, es decir, desde sus propias prácticas de producción de enunciados enseñables. A lo cual Le Moigne (1997) agrega que cuando se reflexiona sobre esas prácticas, se pueden formular algunas hipótesis básicas socioculturalmente aceptables que constituyen uno o varios paradigmas epistemológicos alternativos, al menos tan legítimos como los 
paradigmas positivistas exógenos, sean cartesianos, comtianos, carnapianos o popperianos, que se presentan como los únicos admisibles.

De esta manera, se llega a considerar la importancia que tiene para las ciencias de gestión, su aparente incoherencia epistemológica porque permitió, establecer que la incoherencia no estaba en la disciplina sino en los referentes positivistas con los que se pretendía legitimar. Adicionalmente las ciencias de la gestión al construir nuevos discursos epistemológicos que fueran compatibles, por lo menos con sus prácticas metodológicas más comunes contribuyeron en forma decisiva a la gran renovación paradigmática contemporánea que hoy se evidencia en la reconstrucción de las epistemologías constructivistas (Le Moigne, 1997, 183).

Finalmente y como bien se ha señalado desde los planteamientos anteriores, las ciencias de gestión han experimentado obstáculos históricos para sustentar una identidad epistemológica que legitime su estatus científico, especialmente por los paradigmas positivistas que incluyen los cánones hipotético-deductivos propios de las ciencias naturales.

"Las ciencias de gestión deben navegar entre los obstáculos de la técnica ciega, de la ideología pura, del sofisma generalizado y del cientifismo mutilador. A este precio, las ciencias de la gestión se escapan hacia el grupo de "ciencias verdaderas". Ellas pueden incluso contribuir al renacimiento de los métodos" (Martinet, 1990).

Las ciencias de gestión seguirán entonces en un proceso de construcción permanente de su objeto, métodos y campos de acción, sus postulados, cada vez, irán adquiriendo mayor validez en la medida que se siga reconociendo su pertinencia desde la acción en las organizaciones.

\section{Hacía la gestión de organizaciones}

El campo de conocimiento y por ende de desempeño de la disciplina debe avanzar y trascender del concepto de Administración de Empresas, hacia el de Gestión de Organizaciones. Sin embargo aún se encuentra en un proceso de construcción y consolidación de un discurso que le de sustento a dicha perspectiva. En esta parte del artículo se pretende profundizar en el concepto de organización ${ }^{4}$ como objeto de estudio de la disciplina, y presentar la perspectiva de comprensión de una gestión compleja de organizaciones.

\subsection{Organizaciones}

Alrededor de las organizaciones se han dado numerosas definiciones, provenientes incluso, de diversas ramas del conocimiento, sin que se haya llegado a un consenso en torno a una definición concreta y particular. De acuerdo con Rojot (2005), se pueden identificar algunos temas en común en estas definiciones, como la existencia en una organización de un sistema, objetivos, de la emergencia, vínculos, interacciones, división, reagrupamientos, coordinación, miembros, límites, duración y permanencia, entre otros. El mismo autor propone una segunda aproximación, en la que la organización es una respuesta al problema de la acción colectiva:

"Desde que una actividad no pueda ser llevada a cabo exitosamente por un individuo único, aislado, por el ejercicio de sus propias capacidades, aparece entonces el problema de la acción colectiva, en el cual la organización es la respuesta" (Rojot, 2005, 21).

Es entonces claro que la organización evoca a un sistema social, entendido éste como:

"Un todo integrado por elementos que guardan entre sí una relación recíproca" (Mayntz, 1977, 53).

En el cual se presentan interrelaciones entre seres humanos, y entre estos y los demás componentes de la organización, y entre ésta y su entorno, para dar cumplimiento a diferentes objetivos, fijados específicamente para dicha colectividad. Es justamen-

$4 \quad$ En el aparte 2., se realiza una conceptualización sobre Gestión. 
te a través de la fijación de objetivos, que se diferencian las organizaciones de otras agrupaciones que se encuentran en la sociedad, pues siguiendo al mismo autor:

"Las organizaciones se distinguen de otras partes integrantes de la 'organización social' en que son creadas conscientemente para perseguir un fin" (Mayntz, 1977, 48).

Muchos autores utilizan sin ningún tipo de diferenciación, los términos organización y empresa, cuando ésta última si bien es un tipo de organización, que además cumple con las características propias de las organizaciones, no se puede equiparar a la totalidad de estas. De acuerdo con Mayntz (1997, 49), la empresa "está 'organizada', en el sentido de la teoría económico - empresarial, (pero) su ser no se agota en su 'organización'”, razón por la cual, incluso la formación organizacional conocida como empresa, va mucho más allá de la búsqueda indiscriminada de lucro, pues el concepto de organización, al menos comprendido desde la sociología, designa una formación, no una actividad.

Otro problema que suele encontrarse en torno al concepto de organización, es la confusión que se da al equiparar la organización con la estructura de ésta. Se puede afirmar, que la organización no se limita a la estructura, pues:

"La organización no es un objeto, una cosa invariante independiente de su observador. Expresa a la vez, inseparablemente, la acción, el actor, y la transformación temporal del actor" (Le Moigne, 1999, 76).

Y la estructura suele ser la representación de lo formal en la organización, sin embargo el sistema social en su totalidad, contiene el aspecto informal, pues la estructura es:

"El modelo relativamente estable de la organización; es tan sólo un aspecto del todo y nunca es idéntica al sistema social" (Mayntz, 1977, 105-106).
El componente informal de las organizaciones debe ser tenido en cuenta a la hora de abordar la problemática de las organizaciones. Es decir, para que su análisis sea completo se debe determinar tanto su estructura intencional como su estructura real, pues para poder gestionar la organización esta debe ser comprendida en su totalidad, lo cual no solo incluye la intencionalidad que llevó a la formulación de una determinada estructura, sino que se debe tener en cuenta lo que sucede efectivamente al interior de la organización (relacionado a lo informal), y además, en su interacción con el entorno.

Asumir la posición de las organizaciones como un sistema social, con las implicaciones que esto tiene, hace pensar en unas características propias para las organizaciones. De acuerdo con Navarro (2000, 137) las organizaciones se:

"Caracterizan por su inestabilidad inherente, por la presencia de fenómenos autoorganizados, por la predominancia de procesos de carácter no lineal y por la importancia de elementos de azar en la determinación de sus futuros, futuros, por otra parte, imprevisibles".

Por esta razón, las organizaciones son sistemas alejados del equilibrio, inestables, no lineales, regidos por la imprevisibilidad, por la incertidumbre, características que hacen de las organizaciones un sistema dinámico complejo.

Una de las características más importantes de las organizaciones es su no linealidad: seres humanos, sociedad y organizaciones, son máquinas no triviales. Hasta cierto punto, los seres humanos somos máquinas triviales, porque la vida social (por lo tanto organizacional), así nos lo exige. Sin embargo, la vida social, se encuentra en constante movimiento y sometida a todo tipo de influencias del entorno, y así, los seres humanos, y por ende las organizaciones:

"En momentos de decisión, en los que la máquina se vuelve no trivial: actúa de una manera que no podemos predecir" (Morin, 2001, 116). 
Razón por la cual las organizaciones no son sistemas que respondan de la misma manera a los mismos estímulos. Los eventos tanto internos como externos, afectan a la organización, pero no la determinan.

Es por esto, que en las organizaciones se debe estar preparado para lo inesperado, para la inestabilidad:

"No podemos encerrarnos en el contemporaneísmo, es decir, en la creencia de que lo que sucede ahora va a continuar indefinidamente" (Morin, 2001, 117).

Además, se debe romper con la visión determinista de las organizaciones, es claro que los inevitables intercambios con el entorno, y las perturbaciones propias de éste (no predecibles), van a afectar a la organización, superando los límites de lo esperado. La inestabilidad inherente a las organizaciones evoca al concepto de autoorganización, que hace referencia a una capacidad de las organizaciones sociales, consideradas como sistemas. Hablar de autoorganización significa que el:

"Sistema dispone de capacidades propias para articular nuevos comportamientos en el marco de su identidad y su autonomía. Significa que las organizaciones no están determinadas desde afuera, que su realidad también debe comprenderse desde la óptica de su lógica interna" (Etkin, 2005, 64).

Por lo que no puede asumirse que las organizaciones, tal como se dijo anteriormente, están determinadas desde afuera, pues cada organización obedece a sus propias lógicas y leyes de funcionamiento. En la actualidad, hay posturas que indican un cambio respecto a la visión tradicional, incluyendo, no sólo el aspecto informal en el análisis de la estructura organizacional, sino el reconocimiento de cada organización como un sistema único. $\mathrm{Al}$ respecto, Etkin $(2005,156)$ afirma que:

"Las organizaciones poseen una identidad definida como invariante en el sentido de que su transformación también implica la aparición de una nueva organización".

Entendida esa identidad, como todo aquello que diferencia a una organización de las demás y, que a su vez, se materializa en la estructura que "es entonces la forma concreta que asume una organización en un aquí y ahora concretos".

La estructura actúa como una representación de la naturaleza de la organización, de la identidad y de las particularidades que contiene en sí misma. La estructura, además, responde a unos dominios en particular, que agrupa los elementos que la constituyen:

"a) el dominio de las relaciones entre los participantes de la organización; b) el dominio de los propósitos (intencionalidad de la organización) y, c) el dominio de las capacidades existentes (recursos, memorias, tecnología y cultura)" (Etkin \& Schvarstein, 2005, 59).

Esto implica que se gesten unos subsistemas en las organizaciones que deben, además, interactuar entre sí. Intervenir una organización, implicará actuar sobre el ser humano de la organización, lo cual tiene connotaciones, sobre todo, en la estructura informal de la organización. Se debe igualmente actuar sobre los objetivos que se determinan para la organización, pues estos le dan un matiz particular a la identidad organizacional, y por último, se debe actuar sobre el campo de las capacidades, que estará determinado, por el tipo de organización al cual se haga referencia. La composición de los subsistemas, tiene una relación directa con la naturaleza de las organizaciones, que estará determinada, de acuerdo con Marx (1986, 335), a las relaciones de producción de una sociedad, pues es sobre estas que:

"Se levanta la superestructura jurídica y política y a la que corresponden determinadas formas de conciencia social. El modo de producción de la vida material condiciona el proceso de la vida social".

Teniendo en cuenta esto, se puede establecer que las organizaciones están compuestas por cinco subsis- 
temas, a partir de la clasificación de los sistemas sociales de Parsons (1960), correspondientes a subsistemas técnicos o de producción, de apoyo, de mantenimiento, de adaptación y gerenciales o directivos.

En las organizaciones los subsistemas de apoyo y adaptación, son los encargados de mantener un contacto con el entorno para poder interactuar con éste, y lograr identificar y responder efectivamente a las necesidades identificadas. Por su parte, los subsistemas de mantenimiento actúan:

"Mediando entre las demandas de la tarea y las necesidades humanas para mantener la estructura en funcionamiento" (Dávila, 2001, 249).

Para que a su vez, el subsistema técnico o de producción transforme o procese la energía. Todo el proceso, está mediado por la gestión hecha a través del subsistema gerencial. Queda así en evidencia, cómo los subsistemas se articulan e interrelacionan, para lograr de manera conjunta, el funcionamiento de la organización.

La preponderancia que se le dé a cada uno de esos subsistemas, dependerá, de la identidad de la organización, pues cada organización al obedecer a intencionalidades y finalidades diferentes, deberá estructurar una composición individual única, de acuerdo con sus particularidades, dándole prioridad a algún factor en particular, sin desconocer la existencia de los otros.

La relación entre subsistemas, se da por la intervención de los seres humanos que constituyen la organización. Lo humano no constituye un subsistema, pues teniendo en cuenta la naturaleza misma de la organización -entendida como sistema social-, lo humano es un asunto de orden transversal. La visión limitada del ser humano en la organización, ha llevado a una visión reduccionista de éste, llevando a la negación del ser humano, limitándolo a la categoría de cosa, es decir, se asiste a la cosificación del ser humano en las organizaciones.

La cosificación origina que la interacción del sujeto con la organización, no se dé en términos de una relación sujeto - objeto, sino de una relación objeto
- objeto. Sin embargo, de acuerdo con lo dicho por Morin (2001, 63):

"El sujeto emerge al mismo tiempo que el mundo [...] allí donde un cierto número de rasgos propios de los seres humanos son incluidos en el objeto - máquina".

Reconociendo la existencia del sujeto en su interacción con el objeto. Esta nueva visión del papel del hombre en la organización, rompe con la relación objeto - objeto que se venía dando, a través de la cosificación del ser humano, proponiendo así, un retorno del sujeto como protagonista a la escena de la organización, reconociéndolo como un actor de ésta (Carvajal, 2003; Chanlat, 2006), estando así, en capacidad de incidir en todos los niveles de la organización en su producción, reproducción y transformación. Se asume además, la importancia de la organización como espacio natural para la interacción social entre sujetos, siendo así la organización, un espacio por excelencia para la subjetivación del ser humano.

En ese contexto, se le otorga un carácter sociopolítico a la organización, al reconocer en este espacio un lugar en donde convergen los distintos actores que interactúan con ésta, y que ponen en juego sus necesidades, temores, anhelos, y sueños, para que sean resueltos a través del diálogo y la concertación. Para Etkin $(2003,35)$ la organización es:

"Un conjunto que se integra con el aporte de una diversidad de grupos internos y externos, con sus respectivos intereses, posiciones e influencias".

Aquí, los límites entre el adentro y el afuera se desvanecen, lo que importa son las relaciones que se establecen como eje fundamental para la sostenibilidad. Esta forma de entendimiento comprende tanto a la empresa como a los agrupamientos sociales, es decir, instituciones sin fines de lucro, cooperativas, asociaciones civiles y entes públicos descentralizados.

\subsection{Gestión de organizaciones}

La complejidad, inherente a las organizaciones, hace un llamado a un replanteamiento de preceptos y de 
modos de funcionamiento tradicionales $e$ incluso conduce a una reflexión sobre cómo podría ser su gestión. Es decir, las organizaciones como sistemas dinámicos complejos requieren de un nuevo enfoque para su comprensión y gestión, requieren una gestión compleja. Por tanto, la problemática ligada a la gestión de organizaciones está cada vez más unida a los mecanismos de regulación de sistemas complejos.

Una gestión compleja brinda una perspectiva holística y a la vez diferenciadora de su acción, perspectiva que reconoce la naturaleza e identidad del fenómeno organizacional, y que es consistente con los ambientes cambiantes y regidos por la incertidumbre en los cuales se mueven las organizaciones actuales. La gestión compleja, reconoce la incertidumbre pero no pretende controlarla, por el contrario, hace uso de ella para permitir la generación del sistema. De igual manera, dicha gestión da cuenta de la no linealidad de los procesos que le son propios, que impactan en mayor o menos medida cada uno de los subsistemas (técnicos o de producción, de apoyo, de mantenimiento, de adaptación y gerenciales o directivos) que conforman la organización, procesos que a la vez hacen posible que desde las interacciones e interrelaciones que promueven se construya la organización. La gestión compleja, además, permite la interacción entre lo interno y externo de la organización como dos aspectos que determinan su naturaleza e identidad, aceptando que no existen formulas o recetas mágicas de gestión, por tanto la gestión parte de la compresión de la organización, y se gestiona a partir de la particularidad, de las necesidades inherentes a cada organización.

Cabe señalar, como lo indican varios autores, que las organizaciones se encuentran enfrentadas a una complejidad que califican de complejidad de abundancia porque valora el número considerable de variables en presencia y sus interdependencias (Langrand-Escure \& Thiétart, 1997). En este contexto, la gestión compleja juega un papel relevante, puesto que entra en un universo de interacciones múltiples que provocan otras acciones, en las cuales los efectos sobre la organización son generalmente imprevisibles al inicio. Las organizaciones deben gestionarse considerando sus distintos niveles de complejidad, por ende su identidad, tanto para explicar su funcionamiento como para evaluar las formas de intervención adecuadas a su realidad, realidad donde se cruzan factores relacionados con lo económico, político, social y cultural.

Son ya numerosos los autores (Stacey, 1991; Morin, 2001; Aktouf, 1998; Pascale, 2000; Etkin, 2003; Navarro; 2000, 2002) que han puesto de manifiesto la necesidad de gestionar acorde a los principios de la complejidad, como la dialógica, la recursividad, la autoorganización, la policausalidad entre otros; y la conveniencia de apartarse de formas de gestión tradicionales derivadas del modelo racional y mecanicista con su énfasis en la predecibilidad y el control.

Después de reconocer el carácter de sistema dinámico complejo de las organizaciones se hace difícil pensar en una gestión desde el marco de la dirección y el control, se requiere una gestión que facilite y promueva un desarrollo organizativo hacia formas de mayor complejidad. La gestión de las organizaciones sociales complejas, debe entenderse como una facilitación de procesos (Zimmerman, 1993). De otra forma, la gestión consistirá en poner todas las condiciones para que la auto-organización ocurra y aprovechar luego sus ventajas (Navarro, 2002). Navarro (2000, 141) propone cuatro principios claves para la gestión compleja de organizaciones, los cuales se señalan en la Tabla2.

Para gestionar en la nueva frontera de la complejidad, los gestores en la estrategia, deben priorizar el entendimiento de la organización como un todo interconectado, y no operar y comprender las partes aisladas del mismo; y deben centrarse en los procesos más que en los resultados. Situación que conduce a que se entiende la estrategia como un mecanismo de acción desde la gestión compleja, y se asocie más con la idea de lo flexible o cambiante, que con lo programado o predeterminado, tiene que ver más con el aprendizaje y la adaptación que con la manera de dar respuesta a un plan formalizado. En ese sentido se debe tener claro que aunque la estrategia se refiere a medidas en el futuro de la organización incluye la posibilidad de maniobrar frente a lo imprevisto y lo impensado (Etkin, 2003). 


\section{HACIA UNA EVOLUCIÓN EN EL CAMPO DEL CONOCIMIENTO DE LA DISCIPLINA ADMINISTRATIVA:}

DE LA ADMINISTRACIÓN DE EMPRESAS A LA GESTIÓN DE ORGANIZACIONES

Tabla 2. Principios claves para la gestión compleja de organizaciones según Navarro 5

Crear y expandir complejidad e incertidumbre

Creación permanente de información y de significados

Gestión centrada en los valores nucleares
Si la gestión tradicional de organizaciones aseguraba la importancia de reducir la incertidumbre ambiental y disminuir la complejidad organizativa (perspectiva tradicional de la gestión), la gestión compleja de la organización nos invita a hacer justo lo contrario.

Si acordamos que la organización es un sistema dinámico complejo, podemos pensar en dos actitudes hacia esta situación. En la primera, la gestión tradicional buscaría controlarlo, ahora bien, no por ello consiguen eliminar la incertidumbre propia del sistema y de su entorno. Como segunda actitud, en lugar de pretender el control, se buscan sus utilidades. Aceptando la inestabilidad inherente de la organización, en donde adquiere especial interés una gestión que añada complejidad e incertidumbre. Al hacer esto, se está facilitando la autoorganización espontánea de la organización, ya que al añadir complejidad e incertidumbre se está añadiendo nueva información que el sistema organizativo puede utilizar.

La gestión debe permitir el desarrollo hacia mayores niveles de complejidad en la organización. Una forma de añadir complejidad en la organización puede ser incorporando nuevos detalles, tales como nuevos mercados en los que trabajar, nuevos productos, nuevas cualidades para los productos, etcétera. Cuando los gestores introducen de forma intencionada cambios desestabilizadores a fin de romper los modelos existentes de comportamiento y provocar la aparición de nuevos modelos están reconociendo la importancia de la creación y expansión de la complejidad e incertidumbre como herramienta de gestión (Navarro, 2000, 141).

Este principio, relacionado con el anterior, obedece a una elemental ley física: la segunda ley de la termodinámica. Crear información es generar neguentropía; es decir, contrarrestar la entropía que en todo sistema abierto se produce; contrarrestar la entropía supone escapar al devenir dictado por la segunda ley, es decir, seguir estando vivos.

En relación con la información, la gestión aquí no tiene como tarea el solo procesamiento de la misma. La gestión va más lejos y se enfatiza la importancia de la creación de información. De otra parte, este principio también representa la necesidad de una libre circulación de la información. Sólo de este modo, la información llega a todas partes y serán los grupos autogestionados quiénes la utilicen según sus necesidades.
Apoyar la existencia de fuerzas contrapuestas en la organización y la utilización del conflicto positivo
La gestión debe facilitar y apoyar la existencia de fuerzas opuestas, de opiniones divergentes. De igual manera desde la gestión, es posible utilizar el conflicto para favorecer cambios, constituyéndose en la condición necesaria para que se produzca el progreso en las organizaciones. La clave radica entonces en la gestión que se haga del conflicto (Navarro, 2000).
La gestión por valores se constituye en una gestión más apropiada en entornos de cambio rápido que otros tipos de gestión más clásicos como la dirección por objetivos o la dirección por instrucciones, lo que ha sido claramente puesto de relieve por García y Dolan (1997). Los valores tienen una mayor capacidad de absorber incertidumbre. Con una gestión centrada en los valores nucleares de la organización se permite una mayor tolerancia y puede que hasta una asunción creativa de la complejidad e incertidumbre cada vez más presentes en los entornos empresariales e, incluso, dentro de las propias organizaciones. Además, los valores pueden permitir encauzar las acciones estratégicas de la empresa al proporcionar una visión (Navarro, 2000).

Desde la perspectiva de una gestión compleja, se puede afirmar que junto al abandono de la pretensión de control, y de la eliminación de la incertidumbre, dicha gestión reconoce que el futuro a largo plazo es intrínsecamente impredecible, que a menudo es difícil atribuir un efecto observado a una causa o conjuntos de causas concretas, que las irregularidades en el rendimiento son una propiedad fundamental del sistema (Navarro, 2002).

5 Los autores. 


\section{Reflexión final}

La evolución de la disciplina administrativa ha conducido a la reflexión sobre la forma como se desarrolla la acción de quien gestiona (sujeto), y también se ha hecho preguntas alrededor del objeto, es decir hacia dónde va dirigida la gestión. Dicha evolución ha sido notoria en el último siglo, no sólo en lo que se refiere a la generación de nuevas herramientas, necesarias para la práctica de la gestión, sino también en el proceso de consolidación de un cuerpo teórico propio. En la actualidad hablar de Gestión de Organizaciones implica responde a la evolución que ha tenido la disciplina Administrativa y encontrar un camino pertinente para responder a los nuevos retos del entorno. En un mundo que cambia continuamente, las formas tradicionales de la administración no permiten responder a las exigencias actuales a las que están expuestas las organizaciones. La comprensión de las organizaciones como un todo, en las que cada uno de sus subsistemas, (de finanzas, recursos humanos, mercadeo, producción) son partes interconectadas se convierte en una perspectiva pertinente para su comprensión e intervención, ofrecida por el paradigma de la complejidad.

Es importante reiterar que desde la perspectiva de la complejidad, las organizaciones son sistemas caracterizados por el desequilibrio, las relaciones no lineales y las propiedades emergentes, lo que necesariamente conlleva a una gestión de acuerdo con estas consideraciones, que bien podría denominarse una gestión compleja. En consecuencia, la gestión de organizaciones debe conducir a una propuesta que permita darle respuesta a los retos que trae consigo el reconocimiento de la complejidad del fenómeno organizacional. De alguna manera, esta perspectiva debe conducir a la creatividad e innovación para que a través de estos elementos el futuro de la organización pase de ser anticipado a ser creado, donde no solo se responda a un plan preestablecido sino donde se le dé cabida a lo imprevisto e impensado.

Un gestor de organizaciones en la actualidad, debe tener presente que derivado del nuevo entorno al que se enfrentan las organizaciones, se ha perdido la importancia de los conceptos de orden, estabi- lidad y control frente a los de desorden, conflicto, inestabilidad, diálogo como fuente de estrategias creativas, favoreciendo la autoorganización espontánea. Por tanto, en un entorno no considerado estable y predecible, sino altamente cambiante $e$ impredecible, cada vez se hace más difícil planificar, organizar, dirigir o controlar, por cual los gestores deben aceptar que las capacidades tradicionalmente reconocidas por la Administración se están quedando obsoletas y rezagadas.

En esa medida el paradigma de la complejidad en la Gestión de organizaciones, se traduce en un cambiante marco de relaciones: nuevas relaciones entre directivos y trabajadores del conocimiento; nuevas relaciones con clientes y proveedores; nuevas relaciones con la sociedad a que se sirve (responsabilidad social); nuevas relaciones con el pasado y el futuro (cambios, flexibilidad). Desde esta perspectiva se supera la visión mecanicista y reduccionista, así como la visión parcializada y funcionalista, lo que permitirá estructurar respuestas pertinentes al fenómeno organizacional. Se hace además un llamado a la reflexión sobre los criterios tradicionales que han imperado en la comprensión e intervención de organizaciones.

\section{Referencias}

Aktouf, O. (2003). La Administración de la excelencia: de la deificación del dirigente a la cosificación del empleado El lado inhumano de las organizaciones. Cali: Universidad del Valle.

Aktouf, O. (1998). La administración entre tradición y renovación. Cali: Artes gráficas del valle.

Carvajal, R. (2003). De lo inhumano y lo humanizable en las relaciones de subordinación. Filosofía, historia y sociología del humanismo en la empresa de negocios. El lado inhumano de las organizaciones. Cali: Universidad del Valle.

Chanlat, J. (2006). Ciencias Sociales y Administración. Medellín: Fondo Editorial Universidad EAFIT.

David A, Hatchuel A \& Laufer R. (2000). (coord.) Les nouvelles fondations des sciences de gestión. Paris: Vuibert

Dávila, C. (2001). Teorías Organizacionales y Administración. Bogotá: McGraw Hill.

Delgado, C \& Sotolongo, P. (2006). La complejidad y el nuevo ideal de racionalidad. La revolución contemporánea del saber y la complejidad social. Hacia unas ciencias sociales de nuevo tipo. Buenos Aires: Colección Campus Virtual CLACSO.

Drucker, P. (1975). La gerencia: Tareas, responsabilidades y prácticas. Buenos Aires: El ateneo. 


\section{HACIA UNA EVOLUCIÓN EN EL CAMPO DEL CONOCIMIENTO DE LA DISCIPLINA ADMINISTRATIVA: DE LA ADMINISTRACIÓN DE EMPRESAS A LA GESTIÓN DE ORGANIZACIONES}

Etkin, J. (2003). Gestión de la complejidad en las organizaciones. Las estrategias frente a lo imprevisto y lo impensado. Buenos Aires: Ediciones Granica.

Etkin, J, \& Schvarstein, L. (2005). Identidad de las organizaciones: invarianza y cambio. Buenos Aires: Editorial Paidós.

García, S \& Dolan, S. (1997): La dirección por valores. Madrid: McGraw-Hill.

Kuhn, T. (1962). The structure of scientific revolutions. Chicago: University of Chicago Press.

Langrand, Escure, L, \& Thiétart, R. (1996). Complexité: du vivant au management. Paris: Encyclopedie de Gestion.

Le Moigne, J. (1997). La incoherencia epistemológica de las ciencias de la gestión. Cuadernos de Economía No. 26. Bogotá: Universidad Nacional de Colombia.

Le Moigne, J. (1999). La Modélisation de Systèmes Complexes. Paris: Dunod.

Lorino, P. (1995). El control de gestión estratégico. Barcelona. Marcombo.

Maldonado, C. (2003). Marco teórico del trabajo en Ciencias de la Complejidad y siete tesis sobre la Complejidad. En: Revista Colombiana de Filosofía de la Ciencia, Universidad El Bosque, 4, (8-9): 139 - 154.

Maldonado, C. (2008). Complejidad y Ciencias Sociales desde el aporte de las Matemáticas Cualitativas. Moebio, Universidad de Chile.

Martinet, A. (1990). Epistémologie et sciencies de gestión. Paris: Economica.

Martínez, B. (2005). Las nuevas formas de organización del trabajo: obstáculo para la construcción de una identidad Trabajo y Subjetividad: Entre lo necesario y lo existente. Buenos Aires: Editorial Paidós.

Marx, K. (1986). Contribución a la crítica de la economía política. Buenos Aires: Siglo XXI Editores.

Mayntz, R. (1977). Sociología de la Organización. Madrid: Alianza Editorial.

Morin, E. (2001). Introducción al Pensamiento Complejo (4a reimpresión ed.). Barcelona: Editorial Gedisa.

Morin, E. (2007). La epistemología de la complejidad. El paradigma ecológico en las Ciencias Sociales. Barcelona: Icaria Editorial.
Navarro, J. (2000). Gestión de organizaciones: Gestión del caos. En: Revista Dirección y Organización, Universidad Politécnica de Madrid, 23: 136 - 145.

Navarro, J. (2002). Las organizaciones como sistemas abiertos alejados del equilibrio. Tesis de doctorado, Universidad de Barcelona, España.

Olmedo, E, García, J \& Mateos, R. (2005). De la linealidad a la complejidad: hacia un nuevo paradigma. En: Cuadernos de Estudios Empresariales, 15: 73-92.

Parsons, T. (1960). Estructure and process in modern society. New York: Free Press.

Pascale, R. (2000): Equilibrios al borde del caos. En: Harvard Deusto Business Review. Parte I, 94: 56-63, y Parte II en, 95: 86-93

Pérez, R. (1998). Mission sur les disciplines de gestion. Paris: Ministère de l'Education Nationale; de la recherche et de la technologie.

Piaget, J. (1975). La epistemología de las relaciones interdisciplinarias. Madrid : Editora Nacional.

Rojot, J. (2005). Théorie des Organisations (2e ed.). Paris: Éditions ESKA.

Romero, R. (1998) "El desarrollo el conocimiento y la gestión de organizaciones”. En: Revista Innovar, 11. Bogotá: Universidad Nacional de Colombia, Facultad de Ciencias Económicas.

Sanabria, M. (2007). De los conceptos de administración, gobierno, gerencia, gestión y management: algunos elementos de corte epistemológico y aportes para una mayor comprensión. En: Revista Universidad \& Empresa, Universidad del Rosario, 13: 155 - 193.

Simon, H. (1972). El comportamiento administrativo. Madrid: Aguilar

Stacey, R. (1991). The chaos frontier: creative strategic control for business. Oxford: Butterworth-Heinemann.

Zimmerman, B. (1993): Chaos \& Nonequilibrium: the flip side of strategic processes. En: Organization Development Journal, 11, (1): $31-38$. 



\title{
ANÁLISIS DEL VALOR AGREGADO DEL CONOCIMIENTO. CASO APLICADO EN UNA INSTITUCIÓN DE EDUCACIÓN SUPERIOR*
}

\author{
PAULA ANDREA MOLINA PARRA**, MARTÍN DARÍO ARANGO SERNA ${ }^{* * *}$ \& SERGIO BOTERO BOTERO \\ POLITÉCNICO COLOMBIANO JAIME ISAZA CADAVID - UNIVERSIDAD NACIONAL DE COLOMBIA
}

Recibido/Received/ Recebido: 25/02/20101 - Aceptado/ Accepted / Aprovado: 04/09/2010

\begin{abstract}
Resumen
Este artículo trata el tema del capital intelectual de una organización, el cual es un concepto pocas veces analizado por las empresas, presentando una herramienta practica de valoración del mismo como lo es el Modelo Knowledge Value Added (KVA), del cual se presenta una amplia concepción teórica. Se muestra un caso de estudio aplicado a una empresa del sector educativo colombiano de cómo aplicar dicha metodología para la valoración del capital intelectual y finalmente, a partir de los resultados encontrados en el caso de estudio, se proponen algunas de las políticas estratégicas que se deberían tener en cuenta a futuro para organizar, equilibrar y optimizar la estructura actual de la empresa donde se realizo la aplicación del modelo.
\end{abstract}

Palabras clave: Capital intelectual, modelo KVA, gestión de la organización, caso de estudio.

\section{ANALYSIS OF KNOWLEDGE ADDED VALUE. CASE APPLIED IN A HIGHER EDUCATION INSTITUTION}

\begin{abstract}
This article is about the intellectual capital of an organization, which is a concept that only few times is analyzed by the companies, presenting a practical tool of valuation such Knowledge Value-Added model (KVA), its theoretical conception is presented. A study case is applied to an enterprise from Colombian educative sector, it is about how to apply such methodology to value intellectual capital and finally, parting from the results found in the case, some strategic policies are presented, which should be considered in the future to organize, balance and optimize the current structure of the company where the application model was made.

Key words: Intellectual capital, KVA model, Organization management, case of study
\end{abstract}

Proyecto: Valoración del Capital Intelectual en una empresa Colombiana. Entidad financiadora: Universidad Nacional de Colombia.

* Ms Ingeniería Administrativa, Profesora Asistente, Politécnico Colombiano Jaime Isaza Cadavid, pamolina@elpoli.edu.co.

*** PhD. Profesor Titular Universidad Nacional de Colombia - Sede Medellín, mdarango@unal.edu.co.

PhD. Profesor Asociado Universidad Nacional de Colombia - Sede Medellín, sbotero@unal.edu.co. 